\title{
Inhaltsverzeichnifs des sieben und vierzigsten Bandes, nach den Gegenständen.
}

\section{Reine Mathematik.}

Nr. der

1. A n a 1 y s i s.

4. Nouvelles recherches sur les Covariants. Par Mr. A. Cayley à Londres.

Heft. Seite.

5. Das elliptische Potenzial. Von Herrn Dr. M. G. von Paucker, b. Secr. der

II. 109 kurländischen Gesellschaft für Literatur und Kunst, corr. Mitgliede der Akademie der Wissenschaften zu St. Petersburg. . . . . . . . . . .

7. Über den ersten der von Gau/s gegebenen Beweise des Reciprocitätsgesetzes in der Theorie der quadratischen Reste. Von Herrn G. Lejeune

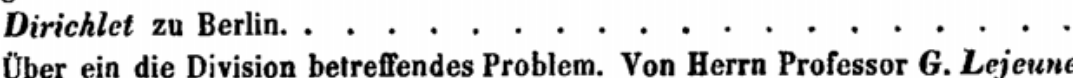

8. Über ein die Division betreffendes Problem. Von Herrn Professor G. Lejeune schaften zu Berlin. Jan. 1851.) . . . . . . . . . . . . . .

9. De formarum binariarum secundi gradus compositione. Auct. G. Lejeune Dirichlet. (Commentatio mense Majo an. MDCCCLI ad actum quendam academicum in univ. litt. reg. Berol. celebrandum typis expressa et distributa.)

11. Über eine Sammlung von bestimmten Integralen. Von Hrn. Bierens de Haan, Oberlehrer der Math. am Gymnasio zu Deventer, math. mag. phil, nat. Doctor.

13. Lösung der Aufgaben $\boldsymbol{C}$. und $\boldsymbol{D}$. in Nr. 21. Band 45 dieses Journals S. 284. Von Dr. phil. Lottuer, Lehrer der Mathematik und Physik an der họhhern Būrgerschule zu Lippstadt. . . . . . . . . . . . . . . . .

16. Méthode du calcul des fonctions elliptiques de troisième espèce. Par Mr. J. Somoff, Professeur à l'Université de St. Petersbourg. . . . . . .

17. Zur Theorie der Abelschen Functionen. Von Dr. C. Weierstrafs, Lehrer der Mathematik am Gymnasio zu Braunsberg in Ostpreufsen. . . . . • III. 269 IV. 289

18. Sur la théorie des formes quadratiques ternaires indéfinies. Par M. Hermite à Paris.

19. Sur la théorie des formes quadratiques. Prennier mémoire. Par le même. IV, 313

20. Sur la théorie des formes quadratiques. Second mémoire. Par le mème. IV. 343

21. Intégration des équations aux différences partielles simultanées d'une certaine classe. Par M. l'abbé Aoust, Professeur de mathématiques pures à la Faculté des sciences de Besançon. 
1. Allgemeine Eigenschaften der algebraischen Curven. Vom Herrn Professor J. Steiner zu Berlin. (Abgedruckt aus dem Monatsbericht der hiesigen Akademie der Wissenschaften vom August 1848.) . . . . . . . .

2. Über solche algebraische Curven, welche einen Mittelpanct baben, und über darauf bezügliche Eigenschaften allgemeiner Curven, so wio über geradlinige Transversalen der letztern. (Theils Auszug, theils Erweiterung eines am 26. Mai 1851 in der Akademie der Wissenschaften gehallenen Vortrags.) Von Demselben.

Heft. Seite.

I. 1

3. Aufgaben und Sâtze, bezüglich auf die vorstehende Abhandlung. Von Demselben. . . . . . . . . . . . . . . . . . . . . .

5. Das elliptische Potenzial. Von Herrn Dr. M. G. von Paucker, b. Secr. der kurländischen Gesellschaft für Literatur und Kunst, corr. Mitgliede der Akademie der Wissenschaften zu St. Petersburg. . . . . . . . . . .

6. Sopra un Teorema di Poligonometria. Nota di P. Tardy, Professore di Matematiche in Genova. (Estratta dagli Annali di scienze matematiche $e$ fisiche pubblicati in Roma Marzo 1852.) . . . . . . . . . . . .

12. Two lettres of the Geometrical correspondence between M. Donkin and M. Spottiswoode.

3. M e c ha n i k.

14. Theorie der Dreh - und Flieh - momente der parallelen Seitenkräfle, in welche Kräfte im Raume zerlegt werden kőnnen. Vom Herrn geh. Rathe und Professor Dr. Schweins in Heidelberg. . . . . . . . . . . . . . III. 238

15. Theorie der Mittelpunkte der parallelen Seitenkräfte. Von Demselben. . . III. 246

\section{Angewand te Mathematik.}

10. An Essay on the Application of mathematical Analysis to the theories of Electricity and Magnetism. By the lato George Green, fellow of Gonvilleand Cains-Colleges at Cambridge. (End of No. 10 tome 39 and No. 22 tome 44.)

Druckfehlerverzeichnifs. . . . . . . . . . . . . . . . . . . IV. 376 